\title{
Disentangling the Mn moments on different sublattices in the half-metallic ferrimagnet $\mathrm{Mn}_{3-x} \mathrm{Co}_{x} \mathrm{Ga}$
}

\author{
P. Klaer, ${ }^{1}$ C.A. Jenkins, ${ }^{2,3}$ V. Alijani, ${ }^{3}$ J. Winterlik, ${ }^{3}$ B. Balke, ${ }^{3}$ C. Felser,${ }^{3}$ and H.J. Elmers ${ }^{1, *}$ \\ ${ }^{1}$ Institut für Physik, Johannes Gutenberg-Universität Mainz, D-55128 Mainz, Germany \\ ${ }^{2}$ Advanced Light Source, Lawrence Berkeley National Laboratory, Berkeley, CA, 94720 \\ ${ }^{3}$ Institut für Anorganische Chemie und Analytische Chemie, \\ Johannes Gutenberg-Universität Mainz, D-55128 Mainz, Germany
}

\begin{abstract}
Ferrimagnetic $\mathrm{Mn}_{3-x} \mathrm{Co}_{x} \mathrm{Ga}$ compounds have been investigated by magnetic circular dichroism in x-ray absorption (XMCD). Compounds with $x>0.5$ crystallize in the $\mathrm{CuHg}_{2} \mathrm{Ti}$ structure. A tetragonal distortion of the cubic structure occurs for $x \leq 0.5$. For the cubic phase, magnetometry reveals a linearly increasing magnetization of $2 x$ Bohr magnetons per formula unit obeying the generalized Slater-Pauling rule. XMCD confirms the ferrimagnetic character with Mn atoms occupying two different sublattices with antiparallel spin orientation and different degrees of spin localization and identifies the region $0.6<x \leq 0.8$ most promising for a high spin polarization at the Fermi level. Individual Mn moments on inequivalent sitesare compared to theoretical predictions.
\end{abstract}

PACS numbers: $61.82 . \mathrm{Bg}, 75.20 . \mathrm{En}, 75.30 . \mathrm{Cr}, 75.50 . \mathrm{Gg}$

This work was supported by the Director, Office of Science, of the U.S. Department of Energy under contract No. DE-ACO2-05CH1123.

DISCLAIMER: This document was prepared as an account of work sponsored by the United States Government. While this document is believed to contain correct information, neither the United States Government nor any agency thereof, nor the Regents of the University of California ("the Regents"), nor any of their employees, makes any warranty, express or implied, or assumes any legal responsibility for the accuracy, completeness, or usefulness of any information, apparatus, product, or process disclosed, or represents that its use would not infringe privately owned rights. Reference herein to any specific commercial product, process, or service by its trade name, trademark, manufacturer, or otherwise, does not necessarily constitute or imply its endorsement, recommendation, or favoring by the United States Government or any agency thereof, or the Regents. The views and opinions of authors expressed herein do not necessarily state or reflect those of the United States Government or any agency thereof or the Regents.

* EMail: elmers@uni-mainz.de 
Heusler compounds are receiving considerable interest due to their predicted half-metallic character.[1, 2] Half-metals have a metallic band structure for one spin channel and an insulating band gap at the Fermi level for the opposite spin thus providing a complete spin polarization of conducting electrons. [3] This is highly advantageous for spintronic applications, where the performance of devices is directly related to the spin polarization of the current emitted and collected by the ferromagnetic electrodes.[2, 4] For applications in spin transfer torque devices, a small magnetization combined with high spin polarization would considerably enhance the effective torque induced by a spin polarized current. [5]

The combination of low magnetization and large local moments can be achieved by the concept of half-metallic ferrimagnetism as proposed by von Leuken and de Groot. [6] Halfmetallic ferrimagnetism has been predicted for $\mathrm{Mn}_{2} \mathrm{VZ}(\mathrm{Z}=\mathrm{Al}, \mathrm{Si})$ [7] and $\mathrm{Co}_{2} \mathrm{TiAl}$ [8] Heusler alloys. Ferrimagnetism has been proven experimentally for $\mathrm{Mn}_{2} \mathrm{VAl}$ and $\mathrm{Co}_{2} \mathrm{TiAl}$ with, however, a very small magnetic moment located at the $\mathrm{V}$ and $\mathrm{Ti}$ atom, respectively. [911] Ferrimagnets with large local moments and half-metallic properties have recently been predicted for compounds that form the $\mathrm{CuHg}_{2}$ Ti-type structure. [12] This crystal structure is based on a cubic structure with four interpenetrating fcc sublattices A, B, C, D [Fig. 1]. In $\mathrm{Mn}_{2} \mathrm{CoGa}$ the Mn atoms occupy sublattices A and B, Co and Ga atoms form sublattices C and D, respectively. In this structure two Mn atoms are in nearest neighbor positions to each other invoking an antiparallel alignment of their magnetic moments. Liu et al. [12] studied the $\mathrm{Mn}_{2} \mathrm{CoZ}(\mathrm{Z}=\mathrm{Al}$, Ga, In, Si, Ge, Sn, Sb) compounds resulting in a mean magnetization that obeys the generalized Slater-Pauling rule. Mn on the A site has a predicted magnetic moment of $-2.2 \mu_{B}$ which is antiparallel to the $\mathrm{B}$ site moment of $+3.3 \mu_{B}$ and the Co moment of $+0.9 \mu_{B}$. Moreover, according to Ref. [13] these compounds possess high Curietemperatures of more than $800 \mathrm{~K}$.

In this work we investigated a series of $\mathrm{Mn}_{3-x} \mathrm{Co}_{x} \mathrm{Ga}$ compounds where the Co atom on the $\mathrm{C}$ site is successively substituted by Mn. Thus the magnetization can be tuned to smaller values. The partial replacement of Co by Mn also avoids an occupation of B sites by Co at high Co concentrations leading to a rapid degradation of the half-metallic properties. X-ray absorption spectroscopy (XAS) reveals element-specific magnetic moments versus composition confirming the theoretical prediction. An analysis of the Mn X-ray magnetic circular dichroism (XMCD) spectra reveals the antiparallel alignment of the itinerant magnetic moment on the A site and the localized moment on the B site [Fig. 3(b)]. The different degree 


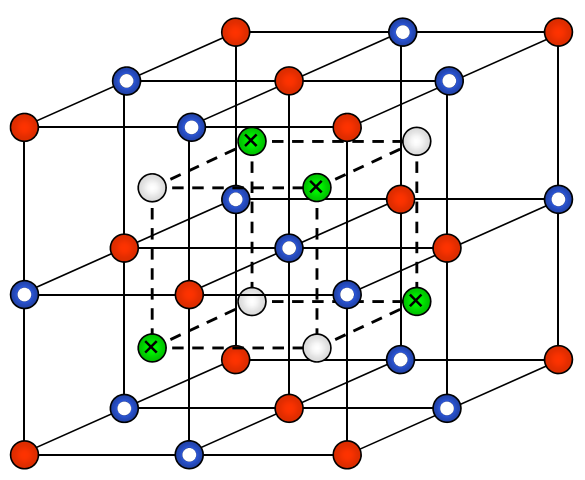

$O A \otimes B O C \bigcirc D$

FIG. 1. (Color online) The generalized cubic structure with four interpenetrating fcc sublattices $\mathrm{A}, \mathrm{B}, \mathrm{C}$, and $\mathrm{D} . \mathrm{Mn}_{3-x} \mathrm{Co}_{x} \mathrm{Ga}$ crystallizes in the $\mathrm{CuHg}_{2}$ Ti-type structure with $\mathrm{Mn}$ occupying the $\mathrm{A}$ and $\mathrm{B}$ sublattice, Co the $\mathrm{C}$ site and Ga the $\mathrm{D}$ site.

of localization as revealed by comparison to spectra from reference compounds agrees well with calculated results [12].

The polycrystalline samples were prepared by arc melting of stoichiometric quantities of the constituents in an argon atmosphere. Oxygen contamination was avoided by evaporating Ti inside the fabrication chamber before melting the constituents, as well as by additional purification of the process gas. The melting procedure was repeated three times to get a homogeneous material. After melting, the polycrystalline ingots were annealed in an evacuated quartz tube at $1073 \mathrm{~K}$ for 8 days. For powder investigations, a part of the sample was crushed by hand using a mortar. The structure was then investigated by x-ray diffraction (XRD) using excitation by Mo-K radiation of powder samples. The magnetic properties were investigated by a super conducting quantum interference device (SQUID). Energy dispersive X-ray spectroscopy (EDX) with an accuracy of \pm 2 -atomic percent was used to check the homogeneity and stoichiometry of the samples.

The XAS/XMCD experiments were performed on beamlines UE56/1 -SGM at the German synchrotron light source (BESSY II) and BL6.3.1 at the Advanced light source (ALS). The samples were cleaved in-situ in UHV directly before the measurement in order to avoid surface contamination. An external field of $2 \mathrm{~T}$ was applied parallel to the incident X-ray beam and perpendicular to the surface and reversed at each $0.1 \mathrm{eV}$ photon energy step.

X-ray diffraction measurements of $\mathrm{Mn}_{3-x} \mathrm{Co}_{x} \mathrm{Ga}$ indicate a transition from the cubic 


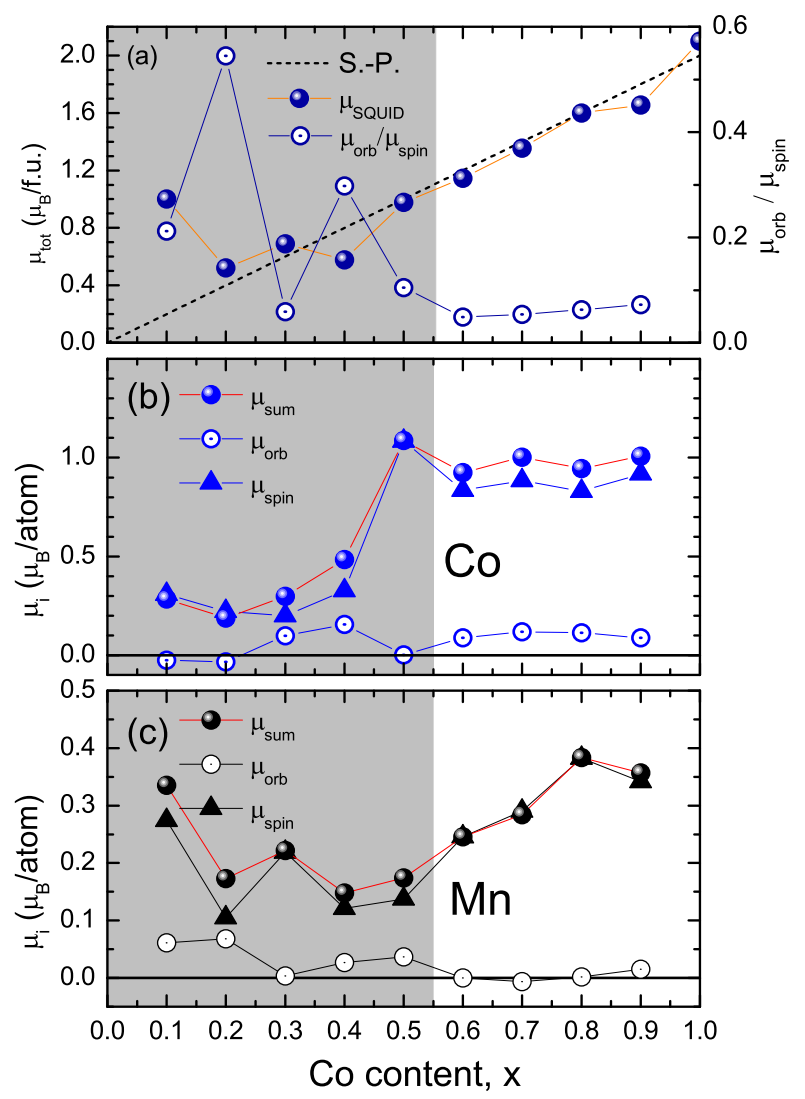

FIG. 2. (Color online) (a) Magnetization (full circles) extrapolated to $20 \mathrm{~K}$ from SQUID magnetometry at room temperature for $\mathrm{Mn}_{3-x} \mathrm{Co}_{x}$ Ga. Open circles denote the orbital to spin moment ratio resulting from the sum rule analysis $[14,15]$. The dotted line indicates the magnetization according to the Slater-Pauling rule. (b) Element-specific spin moments for Co in $\mathrm{Mn}_{3-x} \mathrm{Co}_{x} \mathrm{Ga}$ measured at $20 \mathrm{~K}$ and normalized to the average magnetization as measured by SQUID magnetometry. (c) Similar data for the Mn moments. The shaded area indicates the tetragonally distorted phase.

structure for $x>0.5$ to a tetragonally distorted phase for $x \leq 0.5$. For $x=1$, the formation of the cubic $\mathrm{CuHg}_{2}$ Ti-type structure as shown in Fig. 1 was confirmed in Ref. [12]. The similar scattering factors for the $3 d$ transition elements prevents us from a confirmation of this structure for all values of $x$. The tetragonal phase has been investigated in detail for the case of $x=0$ [16]. This phase cannot be produced without a high degree of disorder and instead of the predicted fully compensated half-metallic ferrimagnet of the cubic phase, the tetragonal $\mathrm{DO}_{22}$ phase leads to a magnetization of roughly $1 \mu_{B}$ per formula unit combined with a reduced spin polarization. 

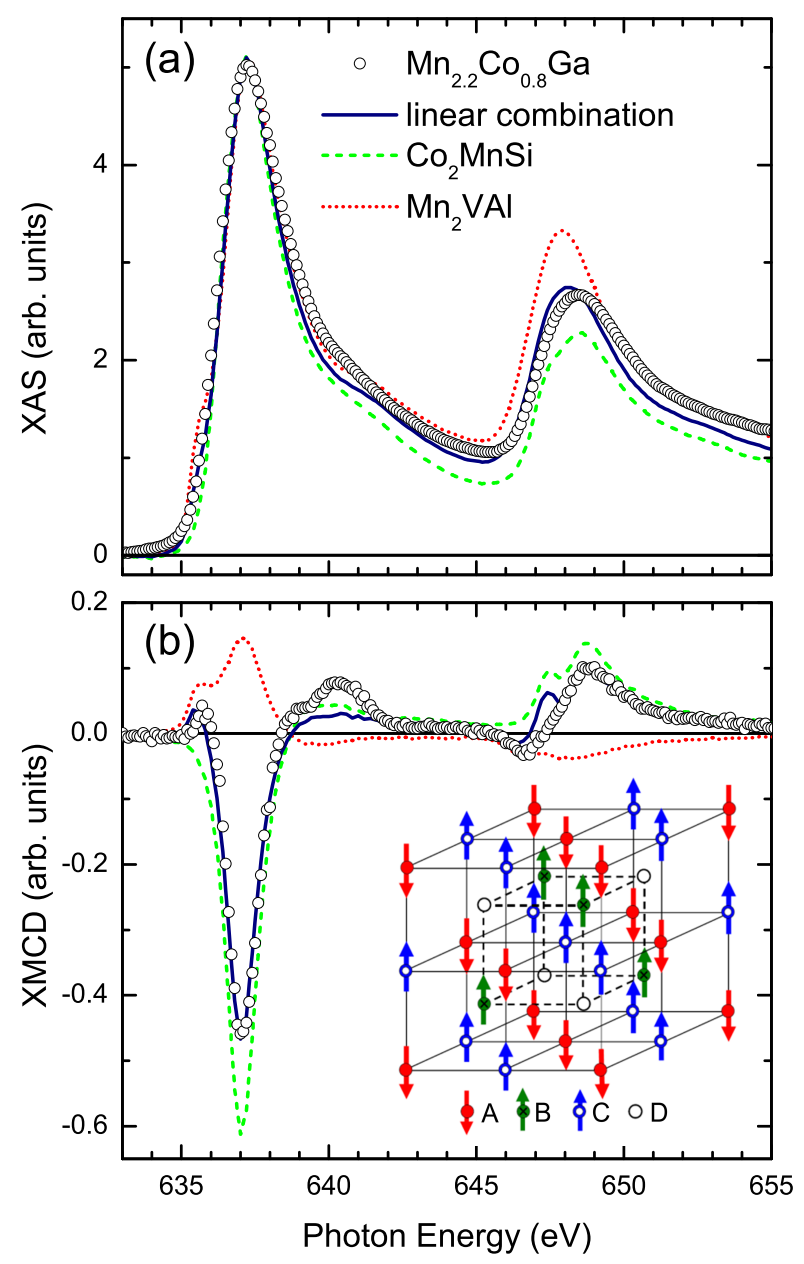

FIG. 3. (Color online) (a) XAS spectra for $\mathrm{Mn}$ in $\mathrm{Mn}_{3-x} \mathrm{Co}_{x} \mathrm{Ga}$ with $x=0.8$ compared to the Mn spectra in $\mathrm{Mn}_{2} \mathrm{VAl}$ [11] and $\mathrm{Co}_{2} \mathrm{MnSi}$ [10]. The thin (green) line indicates the average of both reference spectra. (b) Corresponding data for the XMCD data. The thin (green) line is a linear combination of the reference spectra.

The structural change from cubic to tetragonal with decreasing $x$ considerably changes the magnetic hysteresis. The coercive field rapidly increases for $x \leq 0.5$ indicating a large increase of the magnetic anisotropy for the tetragonal phase. In contrast the deviation of the magnetization from the Slater-Pauling behavior is less sensitive and appears only for $x \leq 0.3$ as shown in Fig. 2 .

Element-specific spin and orbital magnetic moments were determined from XAS/XMCD spectra using the sum rule analysis. For $x>0.5$, we find a good agreement of the total magnetization determined by the sum rules and by SQUID magnetometry, while XMCD results in smaller values for $x \leq 0.5$. We assume that this is caused by the limited external 
field (2 T) thus failing to reach magnetic saturation. Element-specific values shown in Fig. 3 were normalized in order to match the measured magnetization in saturation.

The Co moment increases with its concentration to a maximum value at $x=0.7$ and assumes a nearly constant value of $1 \mu_{B}$ per atom for larger concentrations. In contrast the Mn moment shows a constant up to $x>0.5$. In the range of $0.6 \leq x \leq 0.8$ the Mn moment increases proportional to $x$ as expected for the half-metallic phase. The decreasing Mn moment for high Co concentrations might indicate an increased disorder with Mn-Co antisite defects which would be prohibitive for half-metallic properties. The dependence of the spin moments on the Co concentrations identifies the region $0.6 \leq x \leq 0.8$ most promising for a high spin polarization at the Fermi level.

Interestingly, the orbital magnetic moments of $\mathrm{Mn}$ and Co reveals opposite behavior with increasing Co concentration: For small values of $x$, the Co orbital moment nearly vanishes while Mn has a fairly large orbital moment. For large $x$, the Mn orbital moment approaches zero and the Co orbital moment increases. The decrease of the orbital to spin moment ratio for the summed moments [see Fig. 2(a)] near $x=0.5$ is related to the symmetry increase in the cubic phase.

In order to prove the predicted ferrimagnetism in highly ordered cubic $\mathrm{Mn}_{3-x} \mathrm{Co}_{x} \mathrm{Ga}$ we analyze the Mn XAS/XMCD spectra for $x=0.8$ by comparison with reference spectra from Heusler alloys where $\mathrm{Mn}$ occupies a single sublattice (Fig. 3). $\mathrm{Co}_{2} \mathrm{MnSi}$ is seen as the prototype half-metallic Heusler alloy with a localized Mn moment on the B site in the notation of Fig. 1. The localization of the Mn $d$-bands in this compound causes the multiplet features in the XAS/XMCD spectra that can be simulated quite well using an atomic model. In contrast, the magnetization in $\mathrm{Mn}_{2} \mathrm{VAl}$ originates from itinerant $\mathrm{Mn}$ states occupying the A and $\mathrm{C}$ sublattice according to theoretical and experimental studies. [11]

The linear combination with equal weight of the $\mathrm{Mn}$ spectra from $\mathrm{Co}_{2} \mathrm{MnSi}$ and $\mathrm{Mn}_{2} \mathrm{VAl}$ fits the Mn XAS spectra for $\mathrm{Mn}_{2.2} \mathrm{Co}_{0.8} \mathrm{Ga}$ fairly well (Fig. 3(a)). Prominent features of the $\mathrm{Co}_{2} \mathrm{MnSi}$ spectra namely the $L_{3}$ pre-edge shoulder and the small branching ratio is cut in half, thus indicating the equal occupation of both sites. The small nominal deviation from equal occupation of $\mathrm{A}$ and $\mathrm{B}$ sites by $\mathrm{Mn}$ (because of $x=0.8$ instead of $x=1$ ) cannot be resolved by the fit. The XMCD spectra of $\mathrm{Mn}_{2.2} \mathrm{Co}_{0.8} \mathrm{Ga}$ can also be fitted by a linear combination of the reference spectra [Fig. 3(b)]. In particular the positive $L_{3}$ pre-edge peak is indicative of the presence of antiparallel Mn moments. The deviation at the $L_{2}$ pre-edge 
peak likely originates from a shift of the $\mathrm{Mn}$ multiplet feature from $\mathrm{Mn}_{2.2} \mathrm{Co}_{0.8} \mathrm{Ga}$ to $\mathrm{Co}_{2} \mathrm{MnSi}$ caused by the different crystal field parameters.

Using the values for the averaged Mn moment $\left(\mu_{\text {spin }}=0.4 \mu_{B}, \mu_{\text {orb }}=0.01 \mu_{B}\right)$ per atom and for the ratio of the Mn moment on the $\mathrm{A}$ and $\mathrm{B}$ site $\left(\mu_{\text {spin }}(\mathrm{B}) / \mu_{\text {spin }}(\mathrm{A})=-3.9\right)$ and $\left(\mu_{o r b}(\mathrm{~B}) / \mu_{\text {orb }}(\mathrm{A})=-1.9\right)$ determined from the fit of the reference spectra, individual Mn moments result: $\mu_{\text {spin }}(\mathrm{A})=-0.33 \mu_{B}, \mu_{\text {spin }}(\mathrm{B})=+1.3 \mu_{B}, \mu_{\text {orb }}(\mathrm{A})=-0.03 \mu_{B}$ and $\mu_{\text {orb }}(\mathrm{B})=+0.05 \mu_{B}$. Similar ratios of magnetic moments are observed for $0.7 \leq x \leq 0.9$. The Mn moments are much smaller than values calculated by ab initio theory. This may indicate a residual anti-site disorder that can be sensitively detected by XMCD. For $x<0.7$, the $\mathrm{Mn}$ moment on the A site vanishes completely indicating a strong deviation from the $\mathrm{CuHg}_{2} \mathrm{Ti}$ structure.

In summary, we have demonstrated the preparation of ferrimagnetic $\mathrm{Mn}_{3-x} \mathrm{Co}_{x} \mathrm{Ga}$ compounds with the cubic $\mathrm{CuHg}_{2} \mathrm{Ti}$ structure for $x>0.5$. Disentangling contributions of $\mathrm{Mn}$ atoms on different sublattices reveals fairly large antiparallel oriented Mn moments although the average magnetization is small. The dependence of magnetic moments on the Co concentration identifies the composition $0.6<x \leq 0.8$ most promising for a high spin polarisation at the Fermi level and as a potential candidate for the preparation of highly efficient spin torque devices given that the atomic order can be increased.

We thank S. Cramm for support at BESSY. This project was financially supported by the DFG Research Unit 559, "New Materials with High Spin Polarization" (Germany) and BMBF (Grant No. ES3XBA/5). CAJ acknowledges support of MAINZ, the German Federal Graduate School Of Excellence."

[1] C. Felser, G. H. Fecher, and B. Balke, Angew. Chem. Int. Ed. 46, 668 (2007).

[2] K. Inomata, N. Ikeda, N. Tezuka, R. Goto, S. Sugimoto, M. Wojcik, and E. Jedryka, Sci. Technol. Adv. Mater. 9, 014101 (2008).

[3] J. Kübler, Theory of Itinerant Electron Magnetism, revised ed. (Oxford University Press, New York, 2009).

[4] C. Chappert, A. Fert, and F. Nguyen Van Dau, Nature Mater. 6, 813 (2007).

[5] A. Thiaville, Y. Nakatani, J. Miltat, and Y. Suzuki, Europhys. Lett. 69, 990 (2005). 
[6] H. van Leuken and R. A. de Groot, Phys. Rev. Lett. 74, 1171 (1995).

[7] I. Galanakis, K. Ozdogan, E. Sasioglu, and B. Aktas, Phys. Rev. B 75, 092407 (2007).

[8] S. Ishida, S. Akazawa, Y. Kubo, and J. Ishida, J. Phys. F 12, 1111 (1982).

[9] T. Kubota, K. Kodama, T. Nakamura, Y. Sakuraba, M. Oogane, K. Takanashi, and Y. Ando, Appl. Phys. Lett. 95, 222503 (2009).

[10] P. Klaer, M. Kallmayer, H. J. Elmers, L. Basit, J. Thöne, S. Chadov, and C. Felser, J. Phys. D 42, 084001 (2009).

[11] P. Klaer, E. A. Jorge, M. Jourdan, W. H. Wang, H. Sukegawa, K. Inomata, and H. J. Elmers, Phys. Rev. B 82, 024418 (2010).

[12] G. D. Liu, X. F. Dai, H. Y. Liu, J. L. Chen, Y. X. Li, G. Xiao, and G. H. Wu, Phys. Rev. B 77, $014424(2008)$.

[13] M. Meinert, J.-M. Schmalhorst, and G. Reiss, J. Phys.: Condens. Matter 23, 116005 (2011).

[14] P. Carra, B. T. Thole, M. Altarelli, and X. Wang, Phys. Rev. Lett. 70, 694 (1993).

[15] B. T. Thole, P. Carra, F. Sette, and G. van der Laan, Phys. Rev. Lett. 68, 1943 (1992).

[16] J. Winterlik, B. Balke, G. Fecher, C. Felser, M. Alves, F. Bernardi, and J. Morais, Phys. Rev. B 77, 014424 (2008). 